\title{
Perioperative Indices Predicting Fever Following Percutaneus Nephrolithotomy
}

\author{
Muhammed Cihan Temel ${ }^{1}$, Caner Ediz ${ }^{2}$, Sezgin Okçelik ${ }^{1}$, Halil Kizilöz ${ }^{1}$, Umut Sarioğullari ${ }^{2}$ and Omer Yilmaz ${ }^{2}$ \\ ${ }^{1}$ Department of Urology, Nevsehir State Hospital, Nevsehir, Turkey \\ ${ }^{2}$ Department of Urology, Sultan Abdulhamid Han Education and Research Hospital, Istanbul, Turkey
}

\begin{abstract}
Objective: To investigate 18 independent peroperative parameters that may have an effect on fever after percutaneous nephrolithotomy (PCNL).

Study Design: A descriptive study.

Place and Duration of Study: Departments of Urology, Nevşehir State Hospital, and Sultan Abdulhamid Han Education and Research Hospital, Turkey, from January 2016 to June 2019.

Methodology: Two hundred and seventy-six patients, who underwent PCNL between 2016 and 2019 in two institutions, were evaluated retrospectively. Patients were divided as Group I and II, according to the absence or presence of fever after PCNL, then peroperative parameters were compared.

Results: There was no statistically significant difference between Group I and II in terms of age, gender, side of stone, ipsilateral open stone surgery or PCNL history, presence of renal anatomic anomaly, preoperative hydronephrosis grade, stone size in computed tomography (CT), stone Houndsfield Unit (HU) value, skin stone distance, duration of operation, postoperative D-J insertion rate and postoperative blood transfusion requirement $(p>0.05)$. Guy' s Stone Score (GSS), number of calyceal access and duration of achieving limpid urine coming out of the nephrostomy tube were significantly higher in Group II $(p<0.05)$. The rate of postoperative fever was statistically significantly higher in patients with middle calyceal (mid-calyceal) access, than patients with inferior calyceal access $(p=0.05)$.

Conclusion: High GSS, mid-calyceal access, the increase in the amount of mid-calyceal access, the longer duration of achieving limpid urine coming out of the nephrostomy tube were found to be related with fever after PCNL.
\end{abstract}

Key Words: PCNL, Post-PCNL fever, Nephrolithiasis, Guy's stone score.

How to cite this article: Temel MC, Ediz C, Okçelik S, Kizilöz H, Sarioğullari U, Yilmaz O. Perioperative Indices Predicting Fever Following Percutaneus Nephrolithotomy. J Coll Physicians Surg Pak 2020; 30(12):1306-1311.

\section{INTRODUCTION}

Percutaneous nephrolithotomy is the most recommended treatment option for kidney stones larger than 2 centimetres, according to EAU guidelines. ${ }^{1}$ Moreover, it is one of the preferred treatment options by urologists for multiple and complex kidney stones. ${ }^{2}$

According to current studies, post-PCNL fever may occur in 3.44 to $13.40 \%$ of patients. ${ }^{3,4}$ Many factors associated or not associated with the patient are thought to increase the risk of infection and cause post PCNL fever. ${ }^{5.7}$ Large stone size, staghorn stone and preoperative hydronephrosis grade have been reported as some of the risk factors that may increase complication rates such as fever and sepsis after PCNL. ${ }^{8}$

Correspondence to: Dr. Muhammed Cihan Temel, Department of Urology, Nevsehir State Hospital, Nevsehir, Turkey

E-mail:dr.cihantemel@gmail.com

Received: September 05, 2020; Revised: November 07, 2020;

Accepted: November 17, 2020

DOI: https://doi.org/10.29271/jcpsp.2020.12.1306

Post-PCNL fever is disadvantageous both in terms of patient morbidity and cost-effectiveness. The extra onset of fever after PCNL is a concern for patients, surgeons and the health system. Defining the factors related to fever after PCNL is extremely important for patient management. Previous studies are generally single-centre studies focused mainly on patient-related factors, laboratory tests results, antibiotic use, and the structure of the kidney Stone..$^{5-8}$

If various nomograms can be produced to predict fever after PCNL and algorithms that can be applied earlier in terms of fever management can be developed, they would be a very valuable development for patients, surgeons and the healthcare system.

For these reasons, the aim of this study was to investigate 18 independent factors that may be related to fever formation after PCNL in the light of data collected from both centres.

\section{METHODOLOGY}

Medical records of 317 patients, who underwent PCNL in Nevsehir State Hospital and Sultan Abdulhamid Han Education and Research Hospital, Turkey, from 2016 to 2019 were collected and merged into a conbined dataset. Patients older than 18 years of age, who underwent PCNL surgery and had normal renal anatomy and function, were included in the study. Exclusion criteria were: 
non-consentto participatein the study; age <18years; having solitary kidneys and additional diseases that may affect postoperative fever (positive urine culture, other infections apart from urinary tract, chronic pulmonary disease that may cause ventilation problems, chronic renal disease, uncontrolled diabetes mellitus, immunodeficiency, other systemic pathologies that can cause rheumatological diseases and hyperplexia etc.); having additional pathology that could increase thelikelihood of postoperative bleeding (HighPTZ/INRlevel, anticoagulantdrug use, thrombocytopenia, severe liver / kidney failure, hereditary coagulopathies, etc.). Moreover, patients whose fever was found to be related to a focus other than the urinary system (respiratory tract infection, atelectasis, pneumothorax, etc.) were excluded from the study. A total of 317 patients' data were obtained and 276 patients, who met the study criteria were included in the study.

Before the surgery, routine surgical preparation tests were obtained from all patients (including CT scan). Patients' age, gender, side of stone, ipsilateral open stone surgery or PCNL history, presence of renal anatomic anomaly, preoperative hyronephrosis grade, stone size on CT (determined by measuring the longest diameter of the stone on CT images, and as the sum of the longest diameter of the stones in case of multiple stones), stone Houndsfield Unit (HU) value, Guy' s stone score (Figure 1), ${ }^{9}$ least distance to stone from skin surface, targeted calyx (upper, middle or lower), amount of calyceal access, duration of surgery, duration of achieving limpid urine coming out of the nephrostomy tube, placement of D-J stent and need for blood transfusions were recorded.

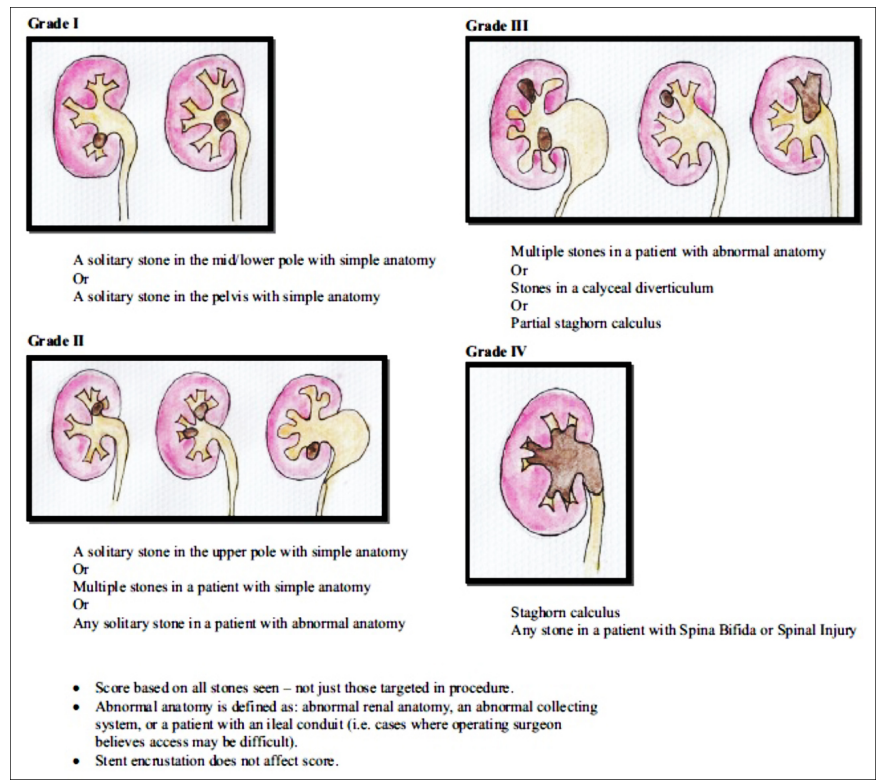

Figure 1: Guy's Stone Score. ${ }^{9}$

Surgeons who participated in the study at both the centres were experienced urologists who have been performing PCNL with same technique for at least 5 years. For these reasons, it was thought that the factors related to the surgeon that might affect the study results were minimised. The operation technique followed these steps: placing a $5 \mathrm{~F}$ or $6 \mathrm{~F}$ open-ended ureter catheter into the ureter, under general anesthesia, under lithotomy position. After the insertion of the ureteral catheter, the patients were placed in prone position. Following surgical sterilisation and patient covering, the collecting system was visualised by retrograde pyelography (RGP). With the $18 \mathrm{G}$ percutaneous access needle, the desired calyx group was targeted and access provided with the help of multiplanar c-arm fluoroscopy at a 90 degrees angle. A 0.038 inch nithinol hydrophilic flexible type guidewire was placed through the access needle into the collecting system. Amplatz dilatation was performed gradually up to $28 \mathrm{~F}$ or $30 \mathrm{~F}$ dilator by starting with $8 \mathrm{~F}$ filiform dilator over the guidewire. Then, the access sheath was placed over the final dilator. Lithotripsy was performed using a $26 \mathrm{~F}$ rigid nephroscope and pneumatic lithotripter. After cleaning the stones, a $14 \mathrm{~F}$ or $16 \mathrm{~F}$ Malecott catheter was placed through the nephrostomy tract to all patients. The integrity of the collecting system and the place of the nephrostomy tube were confirmed by antegrade pyelography. Operation time was calculated as the number of minutes (min.) from the first endoscopic entry to insert a ureteral catheter in the lithotomy position, to the insertion of the nephrostomy tube. Amount of access was calculated as the total number of all access attempts that were successful or failed from the beginning to the end of the operation. In the postoperative period, the time taken until the fluid coming out of the nephrostomy turns to the normal clear urine color was defined as duration of achieving limpid urine coming out of the nephrostomy tube. These durations were calculated by the surgeons during the visits.

The patients were divided into two groups in terms of persistent fever in the postoperative period. Patients without post-op fever were labelled as Group I and with fever were labelled as Group II. At least 2 occurences of $\geq 38 \mathrm{C}^{\circ}$ of fever or $\geq 38 \mathrm{C}^{\circ}$ degrees persistent fever after the first 24 hours postoperatively were accepted as postoperative fever. Chest X-ray was routinely performed in patients with fever. Urinary ultrasonography was performed for urinoma or intra-abdominal extravasation/collection. In patients with persistent fever, $2 \times 1$ gr ceftriaxone treatment was started intravenously. Appropriate antibiotherapy treatment was started with patients who did not respond to antibiotic treatment within 48 hours, by consulting the infectious diseases service, according to the urine and blood culture results.

The statistical analysis of the data was performed using the SPSS version 22.0. Shapiro-Wilk test was used to determine whether the data was normally distributed or not. Qualitative data were expressed as frequencies and percentages. Because the continuous and discrete quantitative data in the study were distributed nonparametrically, the results were reported as Median (IQR). Mann-Whitney U-test, Yates Chi-square (Continuity Correction) test, Fisher's Exact test and Bonferroni corrections were used to compare the groups. Binary logistic regression analysis was used to assess the correlation between the post-PCNL fever and other parameters. Values of $p<0.05$ were considered statistically significant. 
Table I: Median and Inter-Quartile Range (IQR) values of quantitative variables in Group I and II. The numbers of qualitative variables and their percentages in Group I and II. Quantitative/proportional comparison of patients in Group I and II in terms of entered calyx. Group II / group I ratios, according to the entered calixes.

\begin{tabular}{|c|c|c|c|c|c|c|}
\hline & & \multicolumn{2}{|c|}{$\begin{array}{c}\text { Grup I } \\
\text { median (IQR) values }\end{array}$} & \multicolumn{2}{|c|}{$\begin{array}{c}\text { Grup II } \\
\text { median (IQR) Values }\end{array}$} & p-values \\
\hline \multicolumn{2}{|l|}{ Age (years) } & \multicolumn{2}{|c|}{$48(36-58)$} & \multicolumn{2}{|c|}{$35(24-60)$} & 0.105 \\
\hline \multicolumn{2}{|l|}{ Preoperative hydronephrosis grade } & \multicolumn{2}{|l|}{$0(0-2)$} & \multicolumn{2}{|r|}{$2(0-2.5)$} & 0.166 \\
\hline \multicolumn{2}{|l|}{ CT stone size $(\mathrm{mm})$} & \multicolumn{2}{|l|}{$25(20-35)$} & \multicolumn{2}{|c|}{$34(23-43.5)$} & 0.102 \\
\hline \multicolumn{2}{|l|}{ Houndsfield unit } & \multicolumn{2}{|c|}{$1210(1030-1360)$} & \multicolumn{2}{|c|}{$1200(1025-1400)$} & 0.975 \\
\hline \multicolumn{2}{|l|}{ Guy' s stone score (GSS) } & \multicolumn{2}{|c|}{$2(1-2)$} & \multicolumn{2}{|c|}{$2(2-3)$} & $<0.001$ \\
\hline \multicolumn{2}{|l|}{ Skin to stone distance (mm) } & \multicolumn{2}{|l|}{$90(76-105)$} & \multicolumn{2}{|c|}{$72(66-107.5)$} & 0.082 \\
\hline \multicolumn{2}{|l|}{ Duration of surgery (min.) } & \multicolumn{2}{|l|}{$90(60-120)$} & \multicolumn{2}{|c|}{$100(80-125.5)$} & 0.053 \\
\hline \multicolumn{2}{|l|}{ Number of calycial access } & \multicolumn{2}{|l|}{$1(1-1)$} & \multicolumn{2}{|c|}{$1(1-1.5)$} & $<0.001$ \\
\hline \multicolumn{2}{|c|}{ Durations of achieving limpid urine coming out of the nephrostomy (hours) } & \multicolumn{2}{|l|}{$16(8-33)$} & \multicolumn{2}{|c|}{$24(12-47.5)$} & 0.028 \\
\hline & & Group I & Gro & up II & Total & p-values \\
\hline \multirow{2}{*}{ Gender } & Female & $55(19.9 \%)$ & $7(2$ & $5 \%)$ & $62(22.5 \%)$ & 0274 \\
\hline & Male & $200(72.5 \%)$ & $14(5$ & $.1 \%)$ & $214(77.5 \%)$ & 0.214 \\
\hline Side & Left & $132(47.8 \%)$ & $12(4$ & $.3 \%)$ & $144(52.2 \%)$ & 0805 \\
\hline slae & Right & $123(44.6 \%)$ & $9(3$ & $3 \%)$ & $132(47.8 \%)$ & 0.805 \\
\hline & No & $225(81.5 \%)$ & $19(6$ & $.9 \%)$ & $244(88.4 \%)$ & \\
\hline Ipsilateral open stone surgery & Yes & 30 (10.9\%) & 210 & 7\%) & $32(11.6 \%)$ & $>0.999$ \\
\hline Inclateral PCNI hictory & No & $220(79.7 \%)$ & $16(5$ & $.8 \%)$ & $236(85.5 \%)$ & 0203 \\
\hline Ipsilateral PCNL history & Yes & 35 (12.7\%) & $5(1$ & $8 \%)$ & 40 (14.5\%) & 0.203 \\
\hline & No & $249(90.2 \%)$ & $20(7$ & $.2 \%)$ & $269(97.5 \%)$ & \\
\hline Renal anatomic anomaly & Yes & $6(2.2 \%)$ & 110 & $4 \%)$ & $7(2.5 \%)$ & 0.429 \\
\hline & No & $207(75.0 \%)$ & $19(6$ & $.9 \%)$ & $226(81.9 \%)$ & 0386 \\
\hline Postop D-J insertion & Yes & $48(17.4 \%)$ & 210 & $7 \%)$ & $50(18.1 \%)$ & 0.386 \\
\hline Pocton trancfucion & No & $240(87.0 \%)$ & $18(6$ & $.5 \%)$ & $258(93.5 \%)$ & 0147 \\
\hline Postop transtusion & Yes & $15(5.4 \%)$ & $3(1$ & $1 \%)$ & $18(6.5 \%)$ & $0.14 /$ \\
\hline & & & terec & Caly & & Total \\
\hline & & İnferior & Mic & dle & Superior & \\
\hline Group I (Rate to the number of all patients) & & $224(81.2 \%)^{a}$ & $22(8$ & $0 \%)^{\mathrm{b}}$ & $9(3.3 \%)^{a, b}$ & $255(92.4 \%)$ \\
\hline Group II (rate to the number of all patients) & & $13(4.7 \%)^{\mathrm{a}}$ & $7(2$. & $5 \%)^{b}$ & $1(0.4 \%)^{a, b}$ & $21(7.6 \%)$ \\
\hline Grup II / Grup I ratio & & $5.80 \%$ & 31. & $32 \%$ & $11.11 \%$ & $8.23 \%$ \\
\hline p-value & & $p=0.0$ & & & & \\
\hline
\end{tabular}

Table II: Binary regression analysis with post-PCNL fever as the dependent variable and the other parameters as the independent variables.

\begin{tabular}{|c|c|c|c|c|c|}
\hline \multirow{3}{*}{\begin{tabular}{|l} 
Age (years) \\
\end{tabular}} & \multirow{3}{*}{$\begin{array}{c}\boldsymbol{\beta} \text { value } \\
-0.014 \\
\end{array}$} & \multirow{3}{*}{$\begin{array}{c}\text { p-value } \\
0.437 \\
\end{array}$} & \multirow{3}{*}{$\begin{array}{c}\text { Odds ratio } \\
0.986 \\
\end{array}$} & \multirow{2}{*}{\multicolumn{2}{|c|}{$\begin{array}{c}\begin{array}{c}95 \% \text { confidence interval } \\
\text { for } \beta\end{array} \\
\text { Lower to upper }\end{array}$}} \\
\hline & & & & & \\
\hline & & & & 0.951 & 1.022 \\
\hline Gender & 0.978 & 0.089 & 2.660 & 0.863 & 8.201 \\
\hline Side & 0.391 & 0.459 & 1.478 & 0.525 & 4.160 \\
\hline Ipsilateral open stone surgery & 0.435 & 0.616 & 1.545 & 0.283 & 8.439 \\
\hline Ipsilateral PNL history & -0.100 & 0.886 & 0.905 & 0.233 & 3.519 \\
\hline Renal anatomical anomaly & -0.285 & 0.813 & 0.752 & 0.071 & 8.002 \\
\hline Preop hydronephrosis grade & 0.186 & 0.363 & 1.205 & 0.806 & 1.800 \\
\hline CT stone size $(\mathrm{mm})$ & -0.007 & 0.759 & 0.993 & 0.953 & 1.036 \\
\hline Stone houndsfield unit & 0.001 & 0.259 & 1.001 & 0.999 & 1.003 \\
\hline Guys stone score & 0.514 & 0.096 & 1.672 & 0.913 & 3.063 \\
\hline Skin to stone distance (mm) & -0.010 & 0.420 & 0.990 & 0.968 & 1.014 \\
\hline Entered calyx & -1.179 & 0.074 & 0.308 & 0.084 & 1.120 \\
\hline Number of entered calyx & 0.932 & 0.250 & 2.540 & 0.519 & 12.428 \\
\hline Duration of operation (min.) & 0.006 & 0.530 & 1.006 & 0.987 & 1.025 \\
\hline Nephrostomy fluid clarification time (hours) & 0.005 & 0.631 & 1.005 & 0.983 & 1.028 \\
\hline Postop D-J insertion & 0.937 & 0.312 & 2.553 & 0.415 & 15.686 \\
\hline Postop transfusion & -0.866 & 0.364 & 0.421 & 0.065 & 2.734 \\
\hline
\end{tabular}




\section{RESULTS}

A total of 276 patients were included in the study and the median age was $46.50(35.25-58.00)$ years. Two hundred and fourteen (77.5\%) patients were males and 62 (22.5\%) were females. There was a balanced laterality with 144 (52.2\%) left-sided and $132(47.8 \%)$ right-sided stones. A total of $40(14.5 \%)$ patients had ipsilateral PCNL history and $32(11.6 \%)$ patients had a history of ipsilateral open surgery for kidney stone. Renal anatomic anomaly was present in $7(2.5 \%)$ patients. The median preoperative hydronephrosis grade of the patients was $1(0-2)$. The median stone size in CT was $25(20-35) \mathrm{mm}$, and the median stone HU value was 1205 (1030-1362.25). The median Guy's stone score (GSS) was 2 (1-2). The median skin-stone distance was $90(73.50-105.00) \mathrm{mm}$, and the median duration of operation was 90 (60-120) min. Lower pole access was performed in $237(85.9 \%)$ patients, mid-calyx access was performed in $29(10.5 \%)$, and the superior calyx access was performed in $10(3.6 \%)$ patients. The median number of total calyceal access performed during the operation was 1 (1-1). The median value of the duration of achieving limpid urine coming out of the nephrostomy in the postoperative period was 16 (8-33) hours. A total of 50 (18.1\%) patients underwent D-J insertion, and 18 (6.5\%) patients had postoperative blood transfusion.

Two hundred and fifty-five (92.4\%) patients were included in Group I and 21 (7.6\%) were included in Group II. Chest Xray and necessary tests were routinely performed in patients with fever. Urinary ultrasonography was performed when necessary. Patients whose fever was found to be related to a focus other than the urinary system were excluded from the study. In patients who had fever after PCNL; small peri-nephric collections was not considered as urinoma and they included in the study. The parameters which have no statistically significant difference between the two groups and $p$ values are indicated in Tables. The average GSS values, the total amount of calyceal access and the duration of achieving limpid urine coming out of the nephrostomy in Group II were found to be statistically significant higher than the patients in Group I ( $p<0.05$, Table I). When the patients in Group I and II are compared in terms of calyceal access, the highest rate of fever was observed in patients with mid-calyx access. The probability of postoperative fever was found to be statistically and significantly higher in patients who were operated by mid-calyx access than those who were operated by inferior calyx access $(p=0.05)$. However, there was no statistically significant difference in terms of postoperative fever between the patients who were operated by mid-calyx access and superior calyx access (Table I).

There was no statistically significant correlation between the post-PCNL fever and all other parameters in binary logistic regression analysis (Table II).

\section{DISCUSSION}

Fever after PCNL is one of the most common postoperative complications. ${ }^{3,4}$ Postoperative fever increases both patient morbidity and the length of hospital stay. Accordingly, healthcare costs will also increase. Previous studies have identified many factors that affect fever after PCNL, but these studies are mostly single-center studies that have been interpreted according to laboratory analysis results and focused on antibiotic administration. ${ }^{5-8,10}$ Unlike these studies, it is tried to investigate 18 different and independent parameters other than laboratory results, that may have an effect on fever after PCNL, in the light of data collected from two centres. This study was planned considering that any preoperative or intraoperative finding or any detail in the surgical method may be a more meaningful indicator than medical/laboratory test results, in terms of postoperative fever. This study is superior because it is two-instutional, minimises possible bias, and investigates many independent parameters that can affect postoperative fever.

The complexity and size of the stone is one of the most important parameters in preoperative evaluation. Studies investigating the factors that cause postoperative fever have reported that as the size and complexity of the stone increase, complication rates such as fever after PCNL and the risk of developing SIRS also increase. ${ }^{8,10,11}$ In this study, the median GSS in Group II was statistically and significantly higher than Group I $(p<0.001)$. Its results reporting that stone size, stone complexity and ratio of renal anatomical anomaly were higher in the group with fever, are consistent with previous studies.

When the intraoperative surgical technique is evaluated, there are studies reporting that minimal trauma decreases the possibility of postoperative fever, and the increase in the number of accesses performed to reach the pelvicaliceal system increases the probability of postoperative fever and SIRS. ${ }^{12,13}$ But, these studies are single-centred and the number of cases is relatively smaller than this study. The present results support the fact that the number of accesses performed in PCNL is related to post-op fever, similar to previous studies. The most important factor that provides the minimum number of accesses during surgery is the clinic's and surgeon's experience. Postoperative fever ratio may be lower in experienced clinics and surgeons who perform more frequent PCNL, due to lower number of accesses.

Another factor that causes fever after PCNL is the level of bleeding during the procedure. It has been reported in previous studies that the amount of bleeding during PCNL is an influencing factor in postoperative fever. ${ }^{8,14}$ Prolonged surgery due to bleeding also indirectly increases the possibility of post-PCNL fever. However, laboratory tests are not the only option to evaluate the amount of bleeding. In our study, we determined the time of achieving limpid urine coming out of the nephrostomy as the main criterion to eval- 
uate the amount of bleeding. In the present study, the duration of achieving limpid urine coming out of the nephrostomy in Group II was statistically and significantly higher than Group I $(p<0.05)$. The present results support that bleeding is related with postoperative fever and affects it, similar to the study of Chen and Gonzalez-Ramirez. ${ }^{14}$

Another factor that increases PCNL success is the choice of the right calyx. When the literature is scanned for fever after $\mathrm{PCNL}$, information on the selection of the appropriate calyx is not clear. Some studies have reported that all three calyx structures have no difference in terms of fever development after PCNL. ${ }^{15,16}$ In this study, the rate of postoperative fever in patients performed with middle calyx access was higher than in those performed with superior and inferior calyx access, and statistically and significantly higher than in those performed with inferior calyx access $(p=0.05)$. Considering that the immune response to inflammation created during PCNL will be more in places where vascularisation is concentrated, natural ischemic areas in the lower calyx group may be more appropriate access areas for PCNL.

This study has some limitations, too. These were natural shortcomings due to the retrospective planning. According to the previous studies, it was seen that laboratory results of the patients were generally relevant with postoperative fever. Because authors did not expect to achieve meaningful and new results on this subject, they did not evaluate laboratory parameters in the study. Additionally, since this study is retrospective, the authors could not reach all the data such as stone analysis results, stone culture, composition of stones, the presence of residual stones, punctured urine, Creactive protein, Delta neutrophil index, the immune status of the patients and medical comorbid conditions etc. It was aimed to try to create a comprehensive study by including all the data we could reach. The last limitation is that an evaluation related to sepsis could not be made because no sepsis cases were seen in this study.

In addition to such limitations, it has some strengths. It is a double-centered study conducted with data collected from two different regional hospitals that use the same PCNL technique. Therefore, surgeon-related factors, patient-related factors, geographic and demographic factors, which may affect the results of the study, have less impact on results. In addition, the sample and the context was reflected better, the data was more evenly distributed and the bias was lower. More comprehensive and prospective studies are needed to support the results of our retrospectively designed study. If results similar to this study are found, various nomograms may be produced to predict fever after PCNL; and algorithms may be developed that may be applied earlier in terms of fever management.

\section{CONCLUSION}

High GSS, the high degree of access, the middle calyx access, and the prolonged time in achieving limpid urine coming out of nephrostomy were found to be independent factors that related with fever after PCNL.

\section{ETHICAL APPROVAL:}

This prospective descriptive study was approved by the local Ethics Committee of Nevşehir Hacı Bektaş Veli University (2020.15.186).

\section{PATIENTS' CONSENT:}

Informed consents were obtained from patients to publish the data concerning this study.

\section{CONFLICT OF INTEREST:}

Authors declared no conflict of interest.

\section{AUTHORS' CONTRIBUTION:}

MCT, CE: Contributed to the conception and design of the study.

MCT: Collected the data.

MCT, HK: Drafted and revised the manuscript.

MCT, SO, US, OY: Participated in preparing tables and performing statistical analysis.

\section{REFERENCES}

1. Türk C, Neisius A, Petřík A, Seitz C, Skolarikos A, Thomas K. Urolithiasis: EAU Guidelines. ed. 2020. EAU Guidelines Office, Arnhem, The Netherlands; 2020.

2. Sharbaugh A, Morgan Nikonow T, Kunkel G, Semins MJ. Contemporary best practice in the management of staghorn calculi. Therapeutic Advances in Urology 2019; 11:1-9.

3. Ergani B, Karabicak M, Turk H, Yoldas M, Isoglu CS, Suelozgen $T$, et al. Does increased stone-skin distance due to obesity affect outcomes of percutaneous nephrolitho-tomy? J Urol Surg 2019; 6(4):283-8.

4. Gao X, Lu C, Xie F, Li L, Liu M, Fang Z, et al. Risk factors for sepsis in patients with struvite stones following percutaneous nephrolithotomy. World J Urol 2020; 38(1): 219-29.

5. Amri M, Naouar S, Ben Khalifa B, Hmidi N, Braiek S, ElKamel R. Predictive factors of bleeding and fever after percutaneous nephrolithotomy. Tunis Med 2019; 97(5): 667-74.

6. Demirtas A, Baydilli N, Tombul ST, Demirtas T, Sonmez G. Can neutrophil count, lymhocyte count and neutrophil to lymphocyte ratio predict fever following percutaneous nephrolithotomy in patients without risk factors? J Urol Surg 2020; 7(1):21-6.

7. Yu J, Guo B, Yu J, Chen T, Han X, Niu Q, et al. Antibiotic prophylaxis in perioperative period of percutaneous nephrolithotomy: a systematic review and meta-analysis of comparative studies. World J Urol 2019; 38:1685-700.

8. Chen L, Xu QQ, Li JX, Xiong LL, Wang XF, Huang XB. Systemic inflammatory response syndrome after percutan-eous nephrolithotomy: an assessment of risk factors. Int J Urol 2008; 15(12): 1025-8.

9. Thomas K, Smith NC, Hegarty N, Glass JM. The Guy's stone score-grading the complexity of percutaneous nephrolithotomy procedures. Urology 2011; 78(2): 277-81. 
10. Rashid AO, Fakhulddin SS. Risk factors for fever and sepsis after percutaneous nephrolithotomy. Asian J Urol 2016; 3(2): 82-7.

11. Mandal S, Goel A, Kathpalia R, Sankhwar S, Singh V, Sinha $\mathrm{RJ}$, et al. Prospective evaluation of complications using the modified Clavien grading system, and of success rates of percutaneous nephrolithotomy using Guy's Stone Score: A single-center experience. Indian J Urol 2012; 28(4): 392-8.

12. Korets R, Graversen JA, Kates M, Mues AC, Gupta M. Postpercutaneous nephrolithotomy systemic inflammatory response: a prospective analysis of preoperative urine, renal pelvic urine and stone cultures. J Urol 2011; 186(5): 1899-903.

13. Ramaraju K, Paranjothi AK, Namperumalsamy DB, Chennakr- ishnan I. Predictors of systemic inflammatory response syndrome following percutaneous nephro-lithotomy. Urol Ann 2016; 8(4): 449-53.

14. Gonzalez Ramirez A, Camarena L, Gutierrez-Aceves J, Winston Salem N. Risk factors for fever and sepsis after percutaneous nephrolithotomy. J Urol 2013; 189(4S): e633.

15. Caglayan V, Onen E, Avci S, Kilic M, Sambel M, Oner S. Percutaneous Nephrolithotomy via a Middle Calyx Access Is Effective in the Treatment of Lower Pole Kidney Stones: A Single-Center Study. Urol Int 2020; 21:1-5.

16. Deger M, Izol V, Ok F, Bayazit Y, Satar N, Aridogan IA. Comparison of Efficacy and Safety of Isolated Single Different Calyx Accesses in Percutaneous Nephrolithotomy. J Urol Surg 2019; 6(4):289-94. 